\title{
EXPANSIÓN DE LA SOJA EN MÉXICO Y EXCLUSIÓN PRODUCTIVA DE LOS PEQUEÑOS AGRICULTORES DE LA PENÍNSULA DE YUCATÁN
}

\author{
Flavia Echánove Huacuja ${ }^{l}$ \\ Universidad Nacional Autónoma de México
}

\begin{abstract}
RESUMEN
En la península de Yucatán se ha dado un reciente proceso de expansión del cultivo de la soja sobre tierras anteriormente dedicadas a maíz, pastizales y selvas tropicales. Este artículo se inscribe en el debate sobre la "teoría del acceso", planteándose como objetivos la descripción y análisis de las causas o factores que han motivado dicha expansión, así como de sus impactos en el mercado de tierras e ingreso de los productores. También se reseñan los efectos en el medio ambiente (deforestación) y la economía de los apicultores. La investigación reveló cómo una elite de productores y agro-empresas han tenido la habilidad de ganar, controlar y mantener el recurso tierra, a través de diversos mecanismos de acceso, mientras que los pequeños productores (propietarios legales) se han convertido en rentistas de sus tierras, ubicándose en una situación de "exclusión productiva".
\end{abstract}

Palabras clave: soja; exclusión productiva; teoría del acceso; Península de Yucatán; México.

\section{SOYBEAN EXPANSION IN MEXICO AND THE PRODUCTIVE EXCLUSION OF SMALL FARMERS IN THE YUCATAN PENINSULA}

\section{ABSTRACT}

In the Yucatan peninsula there has been a recent process of expansion of soybean cultivation, on lands previously dedicated to maize and grasslands, as well as tropical forests. This article is part of the debate on the "access theory", setting out as objectives the description and analysis of the causes or factors that have motivated this expansion, as well as its impacts on the land market and income of producers. The effects on the environment (deforestation) and the economy of beekeepers are also reviewed. The investigation revealed how an elite of producers and agro-business have had the ability to gain, control and maintain the land resource, through various access mechanisms, while smallholders (legal owners) have become rentiers of their lands, beeing place in a situation of "productive exclusion".

Keywords: soybeans; productive exclusion; access theory; Yucatan Peninsula; Mexico.

1 Instituto de Geografía, Universidad Nacional Autónoma de México, México DF. Correo: flavia2451@gmail.com. Agradezco el financiamiento otorgado por el Programa de Apoyo a Proyectos de Investigación e Innovación Tecnológica (PAPIIT) de esa institución.

Fecha de recepción: 17 de junio de 2020. Fecha de aceptación: 17 de octubre de 2020

Papeles de Geografía, 66 (2020), 68-84 


\section{INTRODUCCIÓN}

Al deforestar los bosques tropicales, cultivar tierras marginales e intensificar la agricultura industrial, la humanidad ha hecho de la agricultura la mayor amenaza para el planeta. Dicho sector productivo ocupa ya un gran porcentaje de la superficie de la tierra y está destruyendo el hábitat, acabando con el agua, contaminando los ríos y océanos y emitiendo gases de invernadero más extensivamente que casi cualquier otra actividad humana (FOLEY, 2011). El reducir estos impactos adversos requiere, entre otras cosas, reflexionar sobre la distribución del suelo agrícola entre cultivos para consumo humano directo y para la fabricación de alimentos animales. Estos últimos utilizan la tercera parte de la producción mundial de granos, y sus superficies cultivadas, junto con la ganadería, ocupan alrededor de tres cuartas partes de la tierra agrícola, emiten hasta $18 \%$ del total de los gases de invernadero y usan 25-32\% del agua, siendo el principal factor de deforestación a nivel mundial (MACHOVINA et al., 2015; HERRERO et al., 2015). La soja (Glycine max) es el grano más utilizado para elaborar alimentos animales, sobre todo para el sector avícola y porcícola, por lo que tres cuartas partes de su producción se destinan a ello. La expansión que la soja ha tenido a nivel mundial responde en gran parte al creciente consumo de carnes, que en las últimas décadas ha tenido un mayor dinamismo en los países en desarrollo, como efecto del incremento de la población y los niveles de ingreso, la urbanización y la adopción de dietas occidentales (WEIS, 2013; HANSEN, 2018).

En el caso de México, la producción de soja durante las últimas décadas solo ha cubierto una mínima parte de su consumo doméstico (5-10\%), por lo que en 2007 el gobierno se planteó disminuir al menos en una tercera parte las compras externas, mediante la promoción de una reconversión productiva para sustituir granos tradicionales (ej. maíz) por oleaginosos (soja, cártamo y canola). Los cuantiosos apoyos o subsidios otorgados desde entonces a la soja, sus elevados precios internacionales, las devaluaciones del peso mexicano frente al dólar estadounidense y el incremento de la demanda, contribuyeron al aumento de las superficies cosechadas, las cuales casi se triplicaron en una década (2008-2018). A pesar de ello, los resultados (190 mil hectáreas cosechadas y 324 mil toneladas producidas) no modificaron el grado de dependencia externa, ya que las importaciones de 2018 (4.8 millones de toneladas) significaron el 94\% del consumo, ubicando a México como el segundo país comprador de soja, después de China (USDA, 2019).

Sin embargo, el aumento de las superficies cultivadas con soja cambió el paisaje de una región particular de México: la península de Yucatán. Ubicada en el sureste del país e integrada por tres entidades (Campeche, Yucatán y Quintana Roo), la península vio aparecer la primera siembra de soja en 2003, a partir de lo cual se expandió sustituyendo pastizales, maíz y selva tropical, a tal grado que las superficies cosechadas entre dicho año y 2018 se incrementaron en poco mas de $8.000 \%$ (de 650 a 55.040 hectáreas). La presente investigación se enmarca en el debate sobre la "teoría del acceso", teniendo como objetivos la descripción y análisis de las causas o factores que han motivado el proceso de expansión de la soja en la península de Yucatán, así como de sus impactos en el mercado de tierras e ingreso de los productores. Dada su relevancia, se reseñan los principales conflictos medio ambientales (ej. deforestación) producto de dicha expansión, así como la afectación a la apicultura de la península. Uno de los hallazgos de la investigación demuestra cómo una elite de productores y agro-empresas, acudiendo a diversos mecanismos de acceso, han tenido la habilidad de ganar, mantener y controlar un recurso natural (tierra), situando a los pequeños productores, garantes de los derechos legales o de propiedad, en una situación de "exclusión productiva". Después de reseñar el marco teórico y métodos utilizados, se analizan las características y relevancia del proceso de expansión de la soja en los tres estados de la península de Yucatán, así como las causas o factores que lo han motivado. Posteriormente, reseñamos algunos de los efectos o impactos más relevantes de dicho proceso, como son la afectación a la cobertura vegetal y a la economía de los apicultores, para concluir con las reflexiones finales de la investigación. 


\section{METODOLOGÍA Y MARCO TEÓRICO}

La metodología utilizada en la investigación consistió en una combinación de métodos cualitativos y cuantitativos (BERG, 2009), priorizando el trabajo etnográfico y la observación participante. Durante 2017-2019 se realizaron 42 entrevistas a profundidad, semi-estructuradas, con informantes claves, en los principales municipios productores de soja de los tres estados de la península de Yucatán. Se entrevistaron a los agentes relevantes que conforman el complejo soja, como son productores y sus organizaciones, tanto del sector social (ejidatarios ${ }^{2}$ ), como del privado (menonitas ${ }^{3}$ y agro-empresas); funcionarios del gobierno federal y estatal; empresas compradoras de soja; distribuidores de semillas (Bayer-Monsanto); y centros de investigación públicos y académicos. Por motivos de confidencialidad, los nombres de todos los entrevistados han sido modificados. La selección de los productores a entrevistar se basó en diversas fuentes, como son los listados de beneficiarios de los programas de apoyo del gobierno a la soja (Agricultura por Contrato y ProOleaginosas) y de los productores que abastecen a las dos empresas compradoras del grano. Para ampliar nuestras redes de informantes, en las visitas a los ejidos y colonias menonitas (productores mayoritarios del grano), acudimos al método de bola de nieve (NADERIFAR et al., 2017). Se visitaron las siete principales colonias menonitas productoras de soja, y los principales ejidos productores y/o rentistas de tierras y sus organizaciones (Unión de Ejidos Emiliano Zapata y Fondo Peto). Las entrevistas se realizaron tanto individual como colectivamente, mediante reuniones convocadas por el representante o gobernador de las colonias, y por las autoridades de los ejidos (comisarios ejidales). Con el fin de conocer la ubicación y dimensión de las superficies rentadas por los menonitas para cultivar soja, se llevó a cabo un ejercicio de mapeo participativo, en el que se pidió a los informantes ubicarlas en un mapa. Esto me permitió conocer los principales ejidos rentistas y dimensionar la expansión de la frontera agrícola.

Tanto en ejidos como en colonias, la información de las entrevistas fue organizada en las siguientes categorías: 1) antecedentes históricos (conformación de los núcleos) y cambios en la producción agrícola (cultivos previos a la soja); 2) causas de dichos cambios (acceso al capital, tecnología, mercados, créditos, apoyos del gobierno, etc.); 3) motivos de la decisión de rentar o tomar en renta las tierras; 4) características de los acuerdos realizados y rentas obtenidas o pagadas; 4) datos del cultivo de soja vs maíz (volumen producido, rentabilidad, comercialización e infraestructura requerida); 5) diversificación de ingresos y percepción de su exclusión productiva (caso de ejidatarios).

Además de la revisión de fuentes periodísticas y publicaciones académicas, en el ámbito cuantitativo se recopilaron estadísticas y reportes oficiales sobre el cultivo de soja en la península (Secretarías de Desarrollo Rural (SADER), Agencia de Servicios a la Comercialización y Desarrollo de Mercados Agropecuarios (ASERCA) y Centros de Apoyo al Desarrollo Rural). Sin embargo, en el caso de Yucatán (región Oriente), la cifra del área cultivada de soja que obtuvimos a través de entrevistas a las dos empresas productoras, resultó el doble de la oficialmente reportada. Fue hasta 2019 cuando la SADER corrigió sus datos.

Para analizar los efectos de la expansión de la soja en las relaciones de producción y el mercado de tierras en la península de Yucatán, nos basaremos en la "teoría del acceso" desarrollada por RIBOT y PELUSO (2009), quienes definen el acceso como la habilidad de beneficiarse de las cosas,

\footnotetext{
${ }^{2}$ Los ejidatarios son los integrantes de los ejidos, los cuales son unidades de producción que se conformaron mediante la distribución de tierras que se dio en México durante la Reforma Agraria, iniciada en 1934. Hasta 1992, en que se modificó la Ley Agraria, los ejidos se organizaban colectivamente y la venta de tierras estaba prohibida, pero a raíz de dicha reforma, los ejidatarios pudieron obtener títulos de propiedad individual y comercializar sus tierras.

${ }^{3}$ Los menonitas constituyen un grupo religioso y étnico que empezó a llegar a México procedentes de Canadá en 1921, estableciéndose inicialmente en el norte del país (TAYLOR, 2005).
} 
incluyendo objetos materiales, personas, instituciones y símbolos. La habilidad incluye, pero no se limita, a las relaciones de propiedad (legal), permitiéndonos entender porqué las personas o instituciones se benefician de los recursos, independientemente de que se tengan o no derechos sobre ellos. Es decir, se plantea priorizar la "habilidad", y no los "derechos" de propiedad (RIBOT, 1998). Metodológicamente, se propone primeramente identificar los beneficios obtenidos, para luego analizar los múltiples mecanismos usados y, finalmente, las relaciones de poder subyacentes. Los mecanismos son el acceso a tecnología, capital, mercados, mano de obra, conocimientos, autoridades, identidades y relaciones sociales. Todos ellos son heurísticos, cada uno de ellos puede permitir, entrar en conflicto o complementar a los otros, resultando en un complejo patrón de distribución de beneficios. Mediante la conformación de "redes de poder", diferentes actores pueden ganar, controlar y mantener los recursos naturales, dependiendo del entorno político-económico y cultural. Como señala PELUSO y RIBOT (2020), es necesario entender el acceso para saber quién se beneficia de los recursos, bajo qué condiciones, movilizando qué relaciones de poder, y a través de qué conjunto de mecanismos.

Los beneficios obtenidos del control de los recursos pueden proceder de la esfera de la producción, extracción, transformación, intercambio, transporte, distribución y consumo, pudiéndose obtener de una sola de esas esferas, de varias o de todas ellas. En el caso del recurso tierra, alguien puede tener la propiedad o el derecho a beneficiarse de ella, pero no poder hacerlo debido a su falta de acceso al capital, tecnología, trabajo, etc. Esto es lo que ha sucedido en la península de Yucatán, donde los pequeños productores (ejidatarios), propietarios legales de las tierras, han tenido que darlas en renta, debido principalmente a su falta de acceso al capital y tecnología necesarios para emprender el costoso e intensivo cultivo de soja. Su conversión en rentistas de tierra a pequeña escala les ha permitido tener cierta participación en los excedentes económicos generados por el complejo soja, pero los ha situado en lo que MCKAY y COLQUE (2016) definen como un estado de "exclusión productiva", en referencia a similar situación de los pequeños productores de Bolivia y la expansión de la soja en ese país. Y es que, de acuerdo a ciertos autores (PELUSO y LUND, 2011; HALL, HIRSCH y LI, 2011), todo el uso y acceso a la tierra implica algún tipo de exclusión.

La elite de productores y agro-industrias que se ha beneficiado de la expansión de la soja en la península de Yucatán ha formado alianzas entre sectores e incrementado su influencia política y económica a través de lo que RIBOT y PELUSO (2009) denominan "relaciones y redes de poder", actuando en concierto con propietarios, trabajadores o beneficiarios, para tener mayor control o mantener el acceso a los recursos. El acceso a la autoridad, definido como el acceso legal e ilegal al estado y otras autoridades, y aplicado ampliamente por MILGROOM y RIBOT (2020), se ejemplifica claramente en los vínculos que la elite de productores de la península ha creado con el gobierno (federal y estatal), lo que les ha permitido, por ejemplo, concentrar los apoyos o subsidios, lograr la legalización de la soja genéticamente modificada (GM) y tener inmunidad ante los daños medio ambientales causados por su actividad. Por su parte, los pequeños propietarios rentistas comparten intereses con los grandes y medianos productores que les arriendan sus tierras, de manera similar a lo encontrado por MCKAY y COLQUE (2016) en Bolivia, entre lo que ellos denominan la "pequeña burguesía rentista" y la clase agro-capitalista.

\section{RESULTADOS}

\subsection{LA EXPANSIÓN DE LA SOJA EN CAMPECHE}

\subsubsection{LOS MENONITAS: PRODUCTORES MAYORITARIOS}

Campeche fue la primera entidad de la península de Yucatán donde se empezó a sembrar soja, registrándose en 2003 las primeras cifras oficiales de su cultivo (650 hectáreas). Sin embargo, para 2018 se cosecharon 41.980 hectáreas de soja, las cuales representaron 76\% del área cosechada de ese 
grano en la península (Tabla 1 y Figura 1). Hopelchén y Campeche son los principales municipios sojeros; el primero de ellos, ubicado en el noreste del estado (región de Los Chenes), en la parte central de la península de Yucatán (Figura 2), es donde más se ha expandido la agricultura mecanizada, y de donde procede la mayoría de la producción estatal de soja (72\% en 2018). Entre los propietarios privados que cultivan el grano destaca el empresario dueño de la firma Proteínas y Oleicos, la compradora mayoritaria de la soja de la península, que en 2018 cosechó 1.730 hectáreas (SADER, 2019). Sin embargo, la gran mayoría de los productores son los menonitas, quienes en 2018 aportaron 90\% de la producción de soja de Campeche (HERNÁN, 2018, entrevista directa). Dicho grupo étnico-religioso arribó a este estado durante los 1980`s, fundando en 1983 la primera colonia menonita de la península de Yucatán (Yalnón), seguida poco después por la colonia Nuevo Progreso (1987) (KAREN, 2019; WISE, 2016, entrevistas directas). Desde entonces, la migración ha sido continua, motivada en gran parte por la búsqueda de tierras para emprender actividades agropecuarias, de tal modo que para 2019 existían alrededor de 2.200 familias menonitas en la península de Yucatán (15.400 personas), ubicadas mayoritariamente en Campeche y, en mucho menor medida, en Quintana Roo. En Yucatán todavía no se han establecido, aunque algunos de ellos ya cultivan tierras en ese estado.

TABLA 1

Península de Yucatán: superficie cosechada y producción de soja (2008-18)

\begin{tabular}{|l|r|r|r|r|r|r|r|r|}
\hline \multirow{2}{*}{ AÑOS } & \multicolumn{2}{|c|}{ CAMPECHE } & \multicolumn{2}{c|}{ YUCATÁN } & \multicolumn{2}{c|}{$\begin{array}{r}\text { QUINTANA } \\
\text { ROO }\end{array}$} & \multicolumn{2}{|c|}{$\begin{array}{r}\text { TOTAL } \\
\text { PENÍNSULA }\end{array}$} \\
\cline { 2 - 10 } & $\begin{array}{r}\text { Sup. } \\
\text { Cos. (Ha) }\end{array}$ & $\begin{array}{r}\text { Prod. } \\
\text { (Ton) }\end{array}$ & $\begin{array}{r}\text { Sup. } \\
\text { Cos. (Ha) }\end{array}$ & $\begin{array}{r}\text { Prod. } \\
\text { (Ton) }\end{array}$ & $\begin{array}{r}\text { Sup. } \\
\text { Cos. (Ha) }\end{array}$ & $\begin{array}{r}\text { Prod. } \\
\text { (Ton) }\end{array}$ & $\begin{array}{r}\text { Sup. } \\
\text { Cos. (Ha) }\end{array}$ & $\begin{array}{r}\text { Prod. } \\
\text { (Ton) }\end{array}$ \\
\hline 2008 & 5.252 & 11.447 & 0 & 0 & 0 & 0 & 5.252 & 11.447 \\
\hline 2009 & 11.208 & 24.457 & 2.775 & 3.893 & 0 & 0 & 13.983 & 28.350 \\
\hline 2010 & 17.660 & 25.229 & 2.384 & 1.333 & 0 & 0 & 20.044 & 26.562 \\
\hline 2011 & 10.727 & 28.879 & 2.037 & 2.099 & 910 & 796 & 13.674 & 31.774 \\
\hline 2012 & 8.143 & 18.616 & 1.221 & 1.674 & 1.216 & 1.736 & 10.580 & 22.026 \\
\hline 2013 & 15.634 & 33.025 & 1.503 & 2.768 & 970 & 1.077 & 18.107 & 36.870 \\
\hline 2014 & 30.366 & 60.602 & 2.089 & 3.637 & 1.856 & 3.712 & 34.311 & 67.951 \\
\hline 2015 & 31.002 & 55.123 & 4.910 & 9.769 & 3.576 & 5.238 & 39.488 & 70.130 \\
\hline 2016 & 37.755 & 96.591 & 8.207 & 19.558 & 2.705 & 3.887 & 48.667 & 120.036 \\
\hline 2017 & 40.020 & 94.842 & 11.071 & 20.668 & 3.050 & 6.216 & 54.141 & 121.726 \\
\hline 2018 & 41.980 & 86.934 & 10.349 & 21.055 & 2.711 & 5.817 & 55.040 & 113.806 \\
\hline
\end{tabular}

Fuente: SIAP, 2019.

Los menonitas se agrupan en "colonias", existiendo actualmente poco más de veinte en Campeche (KAREN, 2019, entrevista directa). La mayoría de las tierras donde se asientan fueron compradas a propietarios privados y/o al gobierno mexicano (tierras federales) (ELLIS et al., 2017), conformándose tanto por espacios habitacionales, como por terrenos aledaños, dedicados a la agricultura hasta donde sus características lo permiten. Las cuatro mayores colonias de la entidad (Nuevo Progreso, Las Flores, La Nueva Trinidad y El Temporal) cultivan entre 5.000 y 12.000 hectáreas cada una, actualmente dedicadas sobre todo a la soja. Su cultivo lo iniciaron en 2003 los menonitas de Las Flores, pero para 2018 diez colonias cosecharon 35.470 hectáreas de soja, lo que 
FIGURA 1

Península de Yucatán: superficie cosechada de soja: 2008-18

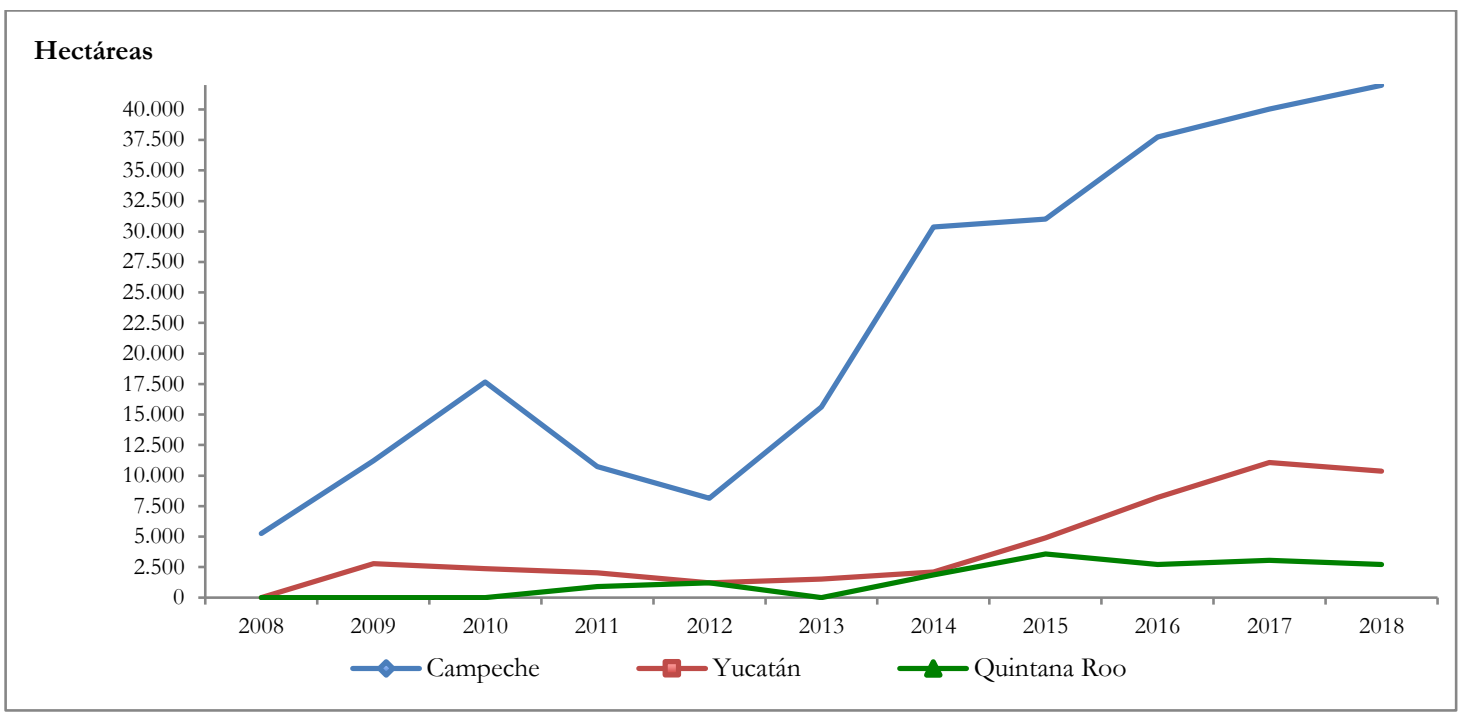

Fuente: Tabla 1.

FIGURA 2

Península de Yucatán: municipios productores de soja (2018)

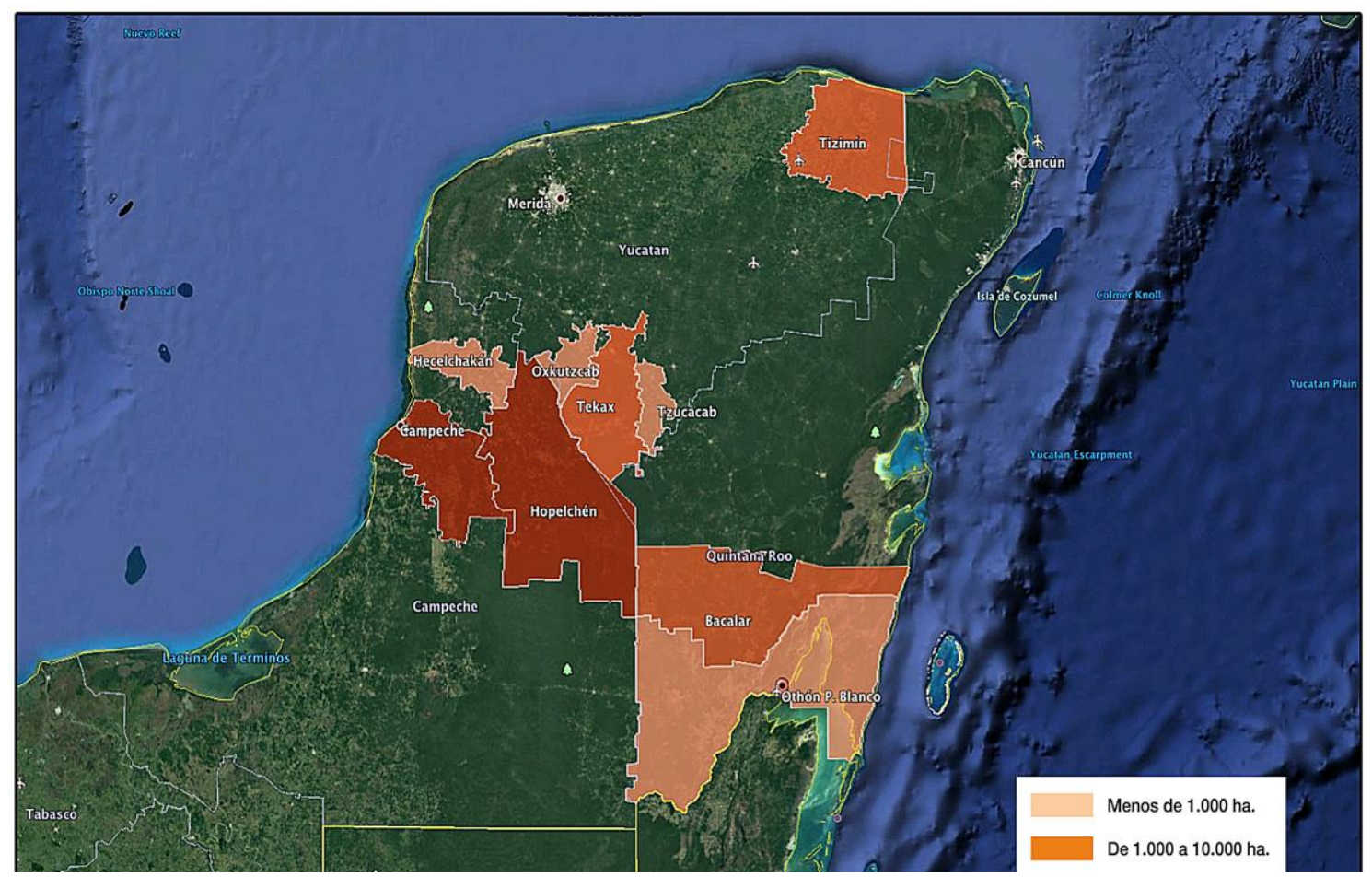

Fuente: GOOGLE EARTH PRO, 2019; GEOCOMUNES, 2019.

Fecha de recepción: 17 de junio de 2020. Fecha de aceptación: 17 de octubre de 2020 
significó $84 \%$ del total de Campeche ${ }^{4}$. El ritmo de expansión de la soja en algunas colonias ha sido impresionante; en 2013, por ejemplo, en la principal colonia, Nuevo Progreso (Figura 3), se cosecharon 500 hectáreas de soja, que aumentaron a 10.000 hectáreas en 2018 (ECHÁNOVE, 2016; LOYD, 2019, entrevista directa). La mayoría de los menonitas cultivan individualmente entre 100 y 400 hectáreas de soja, pero los hay con 800 hectáreas o más. Sin embargo, gran parte de las superficies cultivadas por ellos se encuentran fuera de sus colonias y no son de su propiedad, sino que son tierras rentadas principalmente a ejidatarios $\mathrm{y}$, en menor medida, a propietarios privados, siendo comparativamente reducida la compra-venta de tierras ${ }^{5}$. Las áreas rentadas se ubican sobre todo en Hopelchén, aunque recientemente también en otros municipios más distantes, e incluso en el vecino estado de Yucatán. En 2018, por ejemplo, Nuevo Progreso cultivó 12.000 hectáreas (83\% con soja), integradas por 5.000 hectáreas de su propiedad (ubicadas en la colonia) y 7.000 rentadas en diversos ejidos de la región (PETER, 2019; SAMUEL, 2019, entrevistas directas). Por su parte, los menonitas de La Nueva Trinidad cultivaron alrededor de 8.000 hectáreas (65\% con soja), 4.000 de ellas parte de su colonia y el resto rentadas mayormente a ejidatarios. Actualmente, son muy numerosos los ejidos que rentan la mayoría de sus tierras a los menonitas, situación que ejemplifica claramente lo planteado en la teoría del acceso respecto a que la habilidad de ganar, controlar y mantener los recursos no requiere de su posesión legal o derechos de propiedad (RIBOT y PELUSO, 2009), siendo en este caso la renta de tierras el mecanismo de acceso utilizado. Sin embargo, dicho acceso está teniendo ya claros límites, dado que, de acuerdo a las entrevistas realizadas a menonitas, ya no quedan superficies planas y compactas que puedan rentarse para practicar la agricultura mecanizada (ni en Hopelchén, ni en general en Campeche), es decir, la pérdida de habilidad está determinada por el fin de los recursos a los que se podría acceder.

\subsubsection{EJIDATARIOS PRODUCTORES DE SOJA Y RENTISTAS DE TIERRAS}

La participación de los ejidatarios en la producción de soja de Campeche es muy reducida; en 2018, por ejemplo, alrededor de veinte ejidatarios sembraron 1.800 hectáreas (4\% del total estatal), ubicadas en los ejidos Carlos Cano (CC) y San Luciano. El primer ejido se conformó oficialmente en 1998, cuando 56 ejidatarios fueron dotados cada uno con 150 hectáreas de terreno, 92 de ellas laborables, aunque con cobertura forestal (SUÁREZ, 2005). A partir de la conformación de CC, empezaron a llegar los menonitas, en su mayoría de la cercana colonia de Las Flores, para ofrecer a los ejidatarios desmontar sus tierras con la maquinaria con la que sólo aquellos contaban, a cambio de que les dejasen cultivarlas gratuitamente por 5-6 años, después de los cuales se comprometían a devolverlas. Cuando este lapso terminó, la mayoría de los ejidatarios aceptó la propuesta de los menonitas de tomar en renta las tierras, en las que sembraron primeramente maíz y, a partir de 2007, soja. Los ejidatarios señalan que la falta de maquinaria y capital constituyen los principales impedimentos para emprender el cultivo de soja, el cual, a diferencia del maíz, está completamente mecanizado. Es decir, y de acuerdo a la teoría del acceso, los pequeños productores carecen de dos de los mecanismos de acceso a los recursos, como son el capital y la tecnología (RIBOT y PELUSO, 2009). Por otra parte, el ingreso que obtienen los ejidatarios por rentas es atractivo; en

${ }^{4}$ En 2018, las colonias y sus superficies cosechadas de soja fueron: Nuevo Progreso (10.000 hectáreas), Las Flores (7.400 hectáreas), La Nueva Trinidad (5.200 hectáreas), El Temporal (4.000 hectáreas), Santa Rosa (3.550 hectáreas), Santa Fe (1.800 hectáreas), Las Palmas (1.415 hectáreas), Yalnón (967 hectáreas), Nuevo Durango (900 hectáreas) y Chavi (238 hectáreas) (ORIOL, 2019; LAURO, 2019; LOYD, 2019; PETER, 2019; SAMUEL, 2019, entrevistas directas).

${ }^{5}$ Existe el caso de un grupo de menonitas que compraron tierras en el ejido de Xmabén (municipio de Hopelchén), y que mediante procesos legalmente cuestionados, lograron que en 2008 las tierras del ejido se dividiesen, fundando en parte de ellas el ejido Nuevo Durango (5.656 hectáreas), o sea, se convirtieron en ejidatarios (TORRES et al., 2018). 
FIGURA 3

Colonia menonita Nuevo Progreso (Campeche)

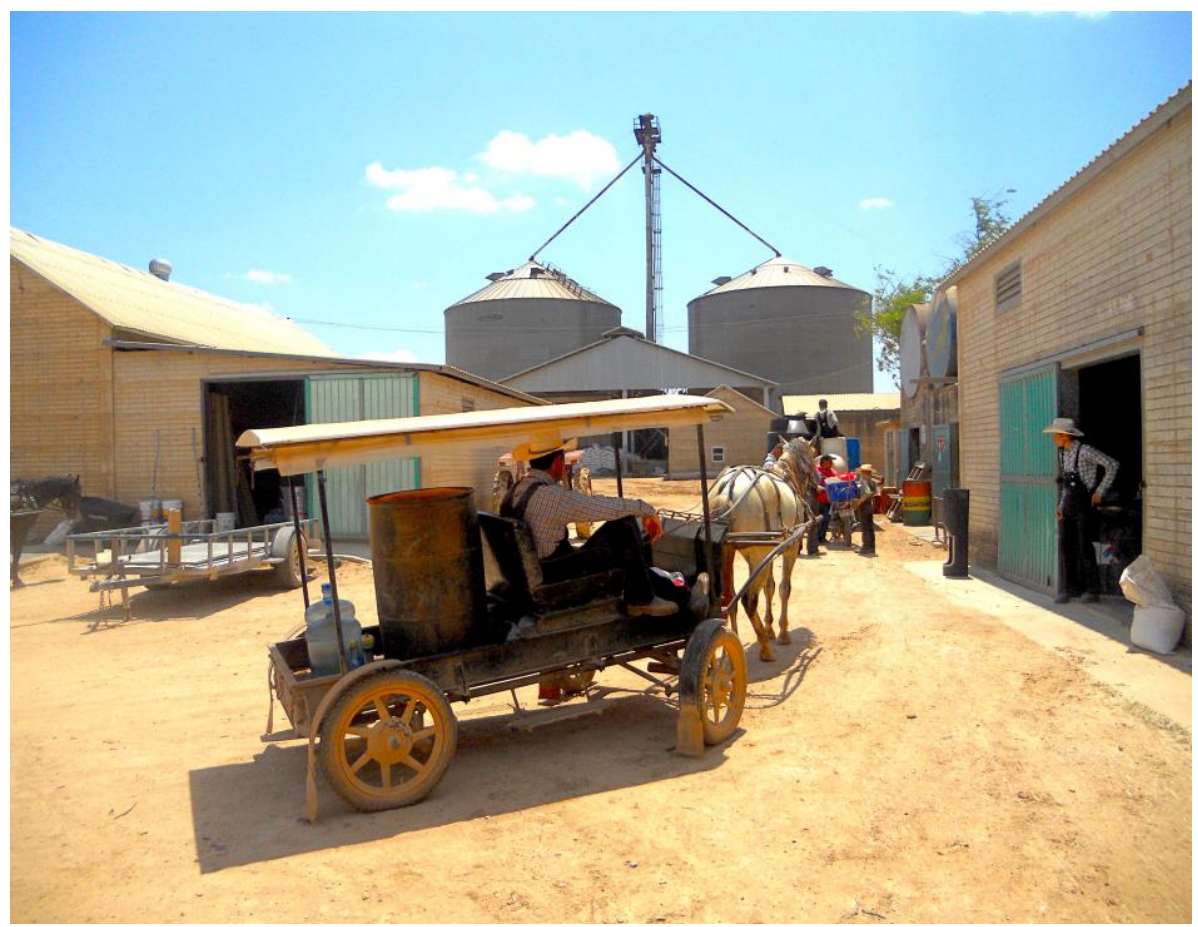

2019, por ejemplo, la hectárea se rentaba en 2.500 pesos mexicanos (MXP) al año, y los contratos entre ejidatarios y menonitas se hacen por lapsos de 2-15 años. Además, el rentar sus tierras les evita asumir el riesgo que todo cultivo implica y les otorga tiempo a ellos y a otros miembros de su familia para dedicarse a otras actividades. Es decir, a pesar de que usualmente las rentas conforman la mayoría de los ingresos de los ejidatarios, éstos ni son los principales beneficiarios del complejo soja, ni pueden insertarse productivamente a él, ya que han sido confinados a una situación de "exclusión productiva", término usado por MCKAY y COLQUE (2016) para calificar similar situación en Bolivia.

Muchos son los ejidos que ejemplifican la magnitud del rentismo de tierras; en el municipio de Hopelchén, por ejemplo, los ejidos Chencoh y Pich rentan 80-90\% de sus tierras a los menonitas de Las Flores. En 2017, uno de los representantes de esta colonia comentó que "toda la sabana está ya rentada" (HANS, 2017, entrevista directa), refiriéndose a la región ubicada al este del municipio de Hopelchén, cercana al límite con Quintana Roo. Recientemente, la renta de tierras para soja se ha expandido incluso hasta la región de La Montaña, la cual forma parte del área de amortiguamiento de la Reserva de la Biósfera de Calakmul y del Corredor Biológico Mesoamericano-México, por lo que se le considera como de alta prioridad para la conservación (PORTER et al., 2008). 


\subsection{LA SOJA EN YUCATÁN: EMPRESARIOS Y EJIDATARIOS RENTISTAS}

El cultivo de soja en Yucatán es más reciente que en Campeche, ya que su siembra inició en 2009, pero entre este año y 2018 las superficies cosechadas aumentaron más del triple (de 2.775 a 10.349 hectáreas), y los volúmenes producidos se quintuplicaron (Tabla 1 y Figura 1). La región sur de Yucatán, conocida por su forma como Cono Sur, fue la primera en cultivar soja, de la cual se cosecharon 5.349 hectáreas en 2018, que significaron poco más de la mitad de las del estado. La mayoría de dicha superficie se localiza en el municipio de Tekax (84\%) (Figura 2), donde los productores privados conforman un reducido grupo que cultiva entre 150 y 300 hectáreas de soja cada uno, entre los que se encuentran algunos menonitas de Campeche. Pero la mayor parte de la soja del Cono Sur es producida en las tierras de 25 ejidos del municipio de Tekax, siendo los más relevantes Huntochac (650 hectáreas de soja), San Felipe (550 hectáreas) y San Isidro Macyam (400 hectáreas). En el primer ejido se dedicaban al cultivo de maíz, ganadería y apicultura, hasta que en 2013 iniciaron un proceso de reconversión de maíz a soja, al igual que lo ocurrido en los otros ejidos mencionados (ECHÁNOVE, 2018).

La producción de soja en el Cono Sur procede de un reducido número de ejidatarios: en 2017, por ejemplo, tres ejidatarios de Huntochac cultivaron $90 \%$ de las superficies de soja en el ejido (CARLOS, 2018; ANTONIO, 2018, entrevistas directas). Esta concentración se ha dado mediante la renta de tierras y, en menor medida su compra, no sólo al interior de Huntochac, sino en ejidos cercanos. Otros ejemplos se dan en San Felipe, el segundo ejido productor, donde cinco ejidatarios concentran las 550 hectáreas de soja que se cultivan, y en San Isidro Macyam (tercer productor), cuyas 400 hectáreas de soja son cultivadas por dos productores. El acaparamiento de tierras en el Sur es de tal magnitud, que se calcula que 15 o 20 productores son los que concentran la producción de soja de la región, a través fundamentalmente de rentas (BRUNO, 2018, entrevista directa). Aunque también existen algunos ejidatarios chicos y medianos que cultivan soja, la gran mayoría de ellos no puede emprender su cultivo por la elevada inversión que se requiere y la falta de maquinaria propia, por lo que optan por rentar sus tierras.

La otra región productora de soja en Yucatán es el Litoral Oriente, concretamente el municipio de Tizimín (Figura 2), donde el cultivo es muy reciente (2013). Sin embargo, entre ese año y 2018 las superficies cosechadas de soja pasaron de 510 a 5.000 hectáreas, convirtiendo a Tizimín en el principal municipio productor Yucatán, y en donde más aceleradamente se ha expandido el cultivo. La región Oriente ha sido históricamente la zona ganadera de Yucatán, albergando $70 \%$ de los pastizales de la entidad y la mayoría de los bovinos existentes (SIAP, 2019). Tizimín concentra más de la mitad de dichos pastizales, parte de los cuales han sido eliminados para cultivar soja, dando lugar a un proceso de reconversión de ganadería a agricultura; sin embargo, la soja también se ha expandido a costa de la selva tropical, causando deforestación. La soja en Tizimín, a diferencia del Cono Sur, es un cultivo completamente empresarial, habiendo dos firmas productoras: EnerAllCargill y El Chilib. La primera fue fundada en 2009 por el empresario mexicano Alfonso Romo, y aunque no se conoce quiénes son los actuales propietarios o accionistas, se ha señalado que un fondo de inversión estadounidense entró a dicha firma en 2015 (ENTREPRENEUR, 2017), del cual Cargill parece ser el socio mayoritario. En 2018, la empresa contaba con 15.000 hectáreas, de las cuales 3.500 se sembraron con soja (BRUNO, 2019, entrevista directa), convirtiéndose en el mayor productor de Yucatán. Debido a que cuentan con riego, establecen dos ciclos productivos al año (soja en primavera-verano, y maíz y sorgo en otoño-invierno), practicándose la siembra directa o labranza cero en la soja. Por su parte, la segunda empresa (El Chilib), fundada por el empresario Jacobo Xacur, empezó en 2011 a comprar tierras en Tizimín, para ganadería y agricultura. Produce maíz y soja, de la cual cosechó 1.500 hectáreas en 2018, todas ellas bajo riego. Sin embargo, su más relevante fuente de ingresos proviene de su empresa Proteínas y Oleicos, la mayor compradora de soja de la península de Yucatán, y uno de los mayores procesadores de este grano en México. Las dos empresas mencionadas concentran $78 \%$ de las áreas irrigadas en la península. 


\subsection{LA SOJA EN QUINTANA ROO: UN CULTIVO DE MENONITAS}

El estado de Quintana Roo ocupa el tercer sitio como productor de soja en la península, y es donde el cultivo de ese grano es más reciente (2011), habiendo sido impulsado por el gobierno estatal mediante el programa Sistema Producto Soja (PIEDRA, 2016). Desde entonces, sus superficies cosechadas se han triplicado (de 910 a 2.711 hectáreas) (Tabla 1 y Figura 1), ubicándose la mayoría de ellas en el municipio de Bacalar (80\% en 2018) (Figura 2), concretamente en el ejido Salamanca, integrado por menonitas. En el otro municipio productor, Othón P. Blanco, las 420 hectáreas cosechadas de soja fueron también cultivadas por menonitas (colonia 4 Banderas) (CHEN, 2019; MORA, 2019, entrevistas directas). El ejido Salamanca es el mayor asentamiento de ese grupo en Quintana Roo, cuyos integrantes migraron de Belice en busca de tierras de cultivo. Ofrecieron al ejido de Bacalar comprar 5.000 hectáreas de sus tierras de uso común, pero dado que éstas eran legalmente inalienables, emprendieron el proceso normativo para convertirse en ejidatarios, para lo cual los 25 menonitas interesados residieron durante un año en el ejido, convirtiéndose así en avecindados, para posteriormente ser reconocidos por la asamblea ejidal como ejidatarios (2005). Esta asamblea también avaló que las 5.000 hectáreas se separasen del ejido Bacalar para ser otorgadas a los menonitas mediante el pago de cierta indemnización. Dado que éstos tenían ya el carácter de ejidatarios, el nuevo núcleo agrario se denominó ejido Salamanca (UH, 2013). Para 2018, se cosecharon 2.216 hectáreas de soja en el ejido, parte de ella GM (PIEDRA, 2016; VILLANUEVA, et al., 2014). El cultivo se lleva a cabo principalmente en las tierras de Salamanca y, en menor medida, en las que los menonitas poseen o rentan en ejidos aledaños. A diferencia de Campeche, en Quintana Roo ha prevalecido la compra de tierras por parte de los menonitas, no sólo vinculadas al cultivo de soja, sino a otros granos como maíz y frijol. La relevante dimensión, presente hasta hoy, de esta comercialización de las tierras ejidales ha sido documentada por diversos autores (MUÑOZ, 2018; HERNÁNDEZ, 2017; CASTILLO, 2017).

\subsection{CAUSAS DE LA EXPANSIÓN DE LA SOJA EN LA PENÍNSULA DE YUCATÁN}

Los determinantes de la expansión de la soja en la península están en gran parte vinculados a la situación del maíz, en el marco de la reconversión productiva habida entre esos dos granos. Incluso en la zona Oriente del estado de Yucatán, donde el cultivo empresarial de la soja se ha expandido sobre todo a costa de los pastizales, las decisiones de los productores han estado determinadas por las ventajas y desventajas entre ambos granos.

\subsubsection{CULTIVO Y GANANCIAS (SOJA VS. MAÍZ)}

Al igual que lo que ocurre en otros países, la soja en la península de Yucatán es mucho más resistente a las sequías que el maíz, lo que constituye una ventaja relevante para los productores, dada la prevalencia de la agricultura de secano en dicha región. Otro factor a favor de la soja han sido sus precios internacionales, los cuales presentaron una tendencia al alza entre 2006 y 2012, año en el que alcanzaron una cifra récord durante décadas (656 USD/ton). En general, dichos precios han sido al menos el doble de los del maíz; en 2018, por ejemplo, el promedio del precio internacional de la soja fue de 356 USD por tonelada, mientras el del maíz ascendió a 164 USD (FAO, 2019) ${ }^{6}$. Estos precios internacionales inciden en los pagados a nivel doméstico; en 2018, por ejemplo, los productores de soja de la península recibieron un promedio de 7.100 MXP por tonelada, cifra que para el maíz fue poco más de la mitad (3.780 MXP) (SIAP, 2019). Y considerando los costos de producción y rendimientos obtenidos por los productores mayoritarios de soja en la península (menonitas de Campeche), sus ganancias son mucho mayores a las obtenidas por el maíz. Para que éste fuese

${ }^{6}$ Precio para la soja amarilla No.1, Fob. Gulf, y para el maíz Fob. Gulf. No. 2, amarillo (FAO, 2019). 
competitivo, es decir, que diese mayores ganancias que la soja, se requeriría obtener un rendimiento de al menos 5 toneladas de maíz por hectárea, cifra muy superior al rendimiento usual de esos productores (3.5 ton/ha).

\subsubsection{SUBSIDIOS DEL GOBIERNO}

Como ya señalamos, desde 2007 el gobierno mexicano empezó a ofrecer diversos apoyos al cultivo de soja, planteándose para el caso de Campeche incrementar en 33.000 hectáreas la siembra de ese grano. Los dos instrumentos utilizados fueron los programas Pro-Oleaginosas (PO), que otorgó a los productores un apoyo monetario por tonelada de soja producida (funcionó hasta 2017), y el programa de Agricultura por Contrato (AXC), el más relevante por la cuantía de los subsidios dados y el número de productores beneficiados. Este programa federal, que protege a productores y compradores de granos de las fluctuaciones de los precios internacionales, empezó a apoyar la soja de la península en 2008, y estaba vigente en 2019, ya con el nuevo presidente Andrés Manuel López Obrador. Esencialmente, el gobierno subsidia el costo de acceder a dicha protección ("primas"), habiendo sido los principales beneficiarios los productores menonitas de Campeche y las empresas más relevantes: Enerall-Cargill (Yucatán), El Yibel (Campeche), El Chilib (Campeche y Yucatán) y Balamte (Campeche) (SADER, 2018), las últimas tres pertenecientes al mismo empresario. Para la gran mayoría de los pequeños productores, el haber quedado excluidos del proceso productivo de la soja los ha eliminado como posibles beneficiarios de la AXC, y los pocos de ellos que cultivan el grano no participan del programa, dadas sus características y complejidad (ECHÁNOVE, 2017).

En la península de Yucatán, el diferencial de precios internacionales (soja vs. maíz) y el mercado seguro para la soja constituían ventajas suficientes a favor de este grano, sin embargo, el gobierno decidió otorgarle cuantiosos apoyos o subsidios, no obstante lo cual no logró reducir el grado de dependencia externa del país, pero sí su meta de expandir el cultivo de soja en la península. Todo esto obliga a reflexionar sobre la política agrícola seguida, que en vez de destinar suficientes apoyos a los pequeños productores de granos básicos (ej. maíz) ha promocionado la agricultura industrial, con graves impactos medio ambientales y económicos.

\subsubsection{LA SEGURA COMERCIALIZACIÓN DE LA SOJA}

La producción de soja de la península es comprada en su totalidad por dos empresas ubicadas en Yucatán: Proteínas y Oleicos (PO) y Crío. La primera produce principalmente pasta de soja ( $80 \%$ del volumen de grano procesado), siendo la quinta productora de ella en el país y la única que la elabora en la península. La gran mayoría del grano que procesa es importado (84\%), siendo los principales compradores de pasta la industria productora de huevo y carne (ave y cerdo) de la península. En 2018, PO compró $94 \%$ de la soja producida en la península, mientras que Crío adquirió el $6 \%$ restante (HERNÁN 2018; BRUNO 2018, entrevistas directas). La segura demanda que estas firmas representan contrasta con la situación del maíz, ya que si bien algunos productores de este grano venden a importantes empresas (harineras, fabricantes de alimentos animales, etc.), la mayoría se enfrenta a problemas de comercialización, sometiéndose frecuentemente al abuso de intermediarios que les ofrecen bajos precios, sabedores de su premura por vender, ya que la mayoría no cuenta con infraestructura de almacenamiento.

\subsubsection{MONSANTO-BAYER Y LAS SEMILLAS GENÉTICAMENTE MODIFICADAS}

Desde que inició el cultivo de soja en la península de Yucatán, se han utilizado tanto semillas de soja GM, como las convencionales (Huastecas), aunque no se cuenta con datos sobre las áreas sembradas con cada una de ellas. Las GM son patrimonio de Bayer, que adquirió Monsanto en 2018, y se conocen como soja Solución Faena (Roundup Ready), resistentes a la aplicación del herbicida glifosato. Desde 1998, Monsanto empezó a producir experimentalmente soja GM en la península de 
Yucatán, pero fue hasta 2012 cuando el gobierno federal concedió el permiso para su cultivo comercial en tres regiones de México, incluyendo la península (PIEDRA, 2016). El gobierno de Yucatán emprendió un litigio en contra de dicha disposición, logrando a fines de 2016 decretar a la entidad como zona libre de cultivos GM; en el caso de la soja, presentó argumentos sobre los efectos negativos del uso del glifosato, sin embargo, en 2019 la Suprema Corte de Justicia anuló dicho decreto, por lo que hoy día el uso de semillas GM en los tres estados de la península es una realidad (TORRES, 2018; CARRERA, 2017; PIEDRA, 2016), como también lo es el papel clave que Monsanto-Bayer ha jugado en la expansión de la soja peninsular, mediante la activa promoción de su cultivo entre los productores, a quienes proporciona asesoría técnica e insumos (semillas y herbicidas).

\section{DISCUSIÓN}

\subsection{APICULTURA Y SELVA TROPICAL: PERDEDORES DE LA EXPANSIÓN DE LA SOJA}

Aunque los efectos del boom de la soja en el medio ambiente y la apicultura de la península no son el objetivo central de nuestra investigación, es relevante hacer ciertos señalamientos. Los efectos adversos del glifosato en la salud (humana y animal), el medio ambiente (contaminación de agua, suelos, etc.) y la biodiversidad, han sido ampliamente documentados (EZQUERRO, 2016; LEGUIZAMÓN, 2016; WHO, 2015; BOHN et al., 2014; GRAIN, 2014; MASON, 2013). En Hopelchén, por ejemplo, se han encontrado residuos de dicho herbicida hasta en el agua potable (RENDÓN et al., 2017). Sin embargo, un hallazgo de mi trabajo de campo fue el constatar que el glifosato es también aplicado en el cultivo de la soja no GM, entre otras razones, porque es el herbicida más económico (a nivel doméstico y mundial). Pero independientemente del tipo de semilla usada, el uso del glifosato, frecuentemente aplicado vía aérea, ha incidido en el incremento de la mortandad de las abejas, las que además entran en contacto con el polen de las flores de la soja GM, ocasionando la presencia en la miel de la península de organismos genéticamente modificados (TORRES, 2018; ELLIS et al., 2017; GÓMEZ, 2016; PIEDRA, 2016; VILLANUEVA et al., 2014). Todo ello ha dañado la economía de los 19.200 apicultores de dicha región, la cual aporta 36\% de la producción de miel de México, el quinto país exportador de ese dulce en 2018 (SIAP, 2019).

Las principales causas de la deforestación en la península han sido la expansión de la ganadería (pastizales), seguida por la agricultura mecanizada (ELLIS et al., 2017; DUPUY et al., 2015), en la que la soja ha sido un factor detonante. Este modelo de agricultura ha sido promovida por el gobierno mediante un cúmulo de incentivos, y se ha expandido sobre todo en el municipio de Hopelchén (Figura 4), el cual perdió 176.000 hectáreas de su cubierta forestal entre 2001 y 2018 (GFW, 2019). Desde mediados de los 1980`s, la mayor tasa de deforestación de dicho municipio se registró durante 2005-2015, ubicándose este proceso principalmente en las propiedades privadas de los menonitas (ELLIS et al., 2017). A estas superficies habría que sumar las deforestadas por dichos agentes en el gran número de ejidos donde rentan tierras, tanto en Hopelchén como en otros, y que, como reveló nuestra investigación, son áreas muy relevantes, escenario sobre todo de la expansión del cultivo de la soja, la cual tuvo su mayor dinamismo justo en dicho período. Un estudio realizado por KELLY (2019) sobre la tasa de deforestación en la región de Los Chenes (a la que pertenece Hopelchén) consideró tres categorías agrarias: ejidos, ejidos que rentan tierras a los menonitas y propiedades privadas de los menonitas, encontrando que durante 2000-2010 las tasas de cambio de la cubierta forestal fueron de $-0.19 \%,-0.47 \%$ y $-1.25 \%$, respectivamente. Es claro que una década después, el porcentaje de los ejidos rentistas debe ser mucho mayor, y sería interesante hacer similar estudio en el municipio de Campeche, donde la renta de tierras a ejidos es también muy relevante.

En el municipio de Hopelchén, la frontera agrícola de los menonitas se ha extendido hasta su parte sur, con una topografía más irregular (lomeríos), y donde se ubica la zona de amortiguamiento de la Reserva de la Biósfera de Calakmul, la mayor área natural protegida de México. Sin embargo, dentro 
de ella (ejido Xmabén) los menonitas han comprado 5.656 hectáreas para el cultivo de granos, incluida soja. El consecuente desmonte de tierras con cobertura forestal, muchas veces

FIGURA 4

Campeche: cambio de uso del suelo y vegetación (1976-2016)
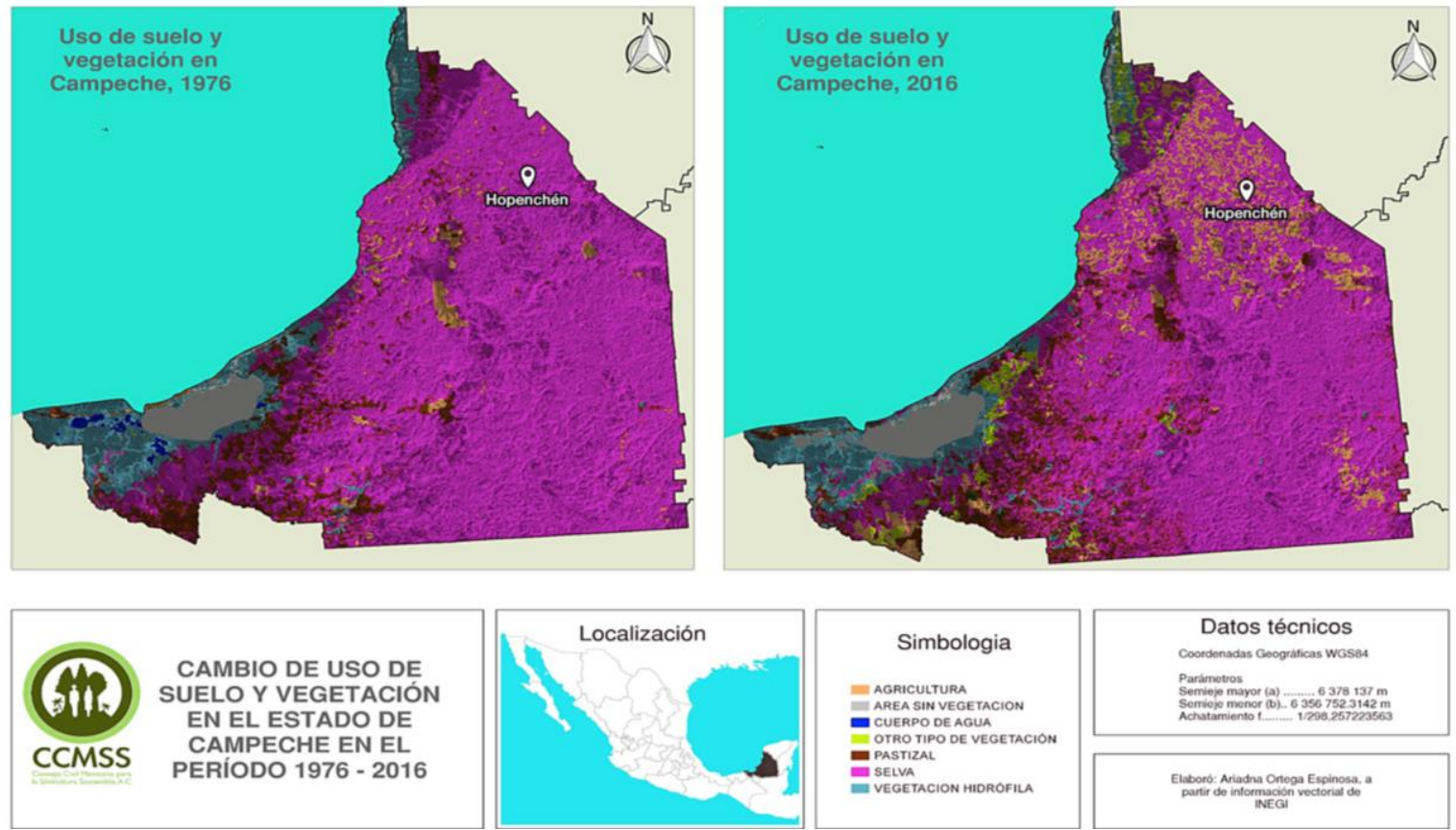

Fuente: CCMSS, 2019.

ilegal, se ha dado también en otras zonas del estado de Campeche, así como en Quintana Roo, donde los menonitas han deforestado 2.600 hectáreas, de las cuales 300 pertenecían a áreas naturales protegidas ubicadas en los municipios de Bacalar y José María Morelos (ESTOS DÍAS, 2019). En ese mismo estado, se ha documentado con imágenes el proceso de deforestación habido en el ejido menonita de Salamanca (principal productor de soja), que ha ocasionado la pérdida del $66 \%$ de la cobertura forestal entre 2002 y 2013, afectando a una de las masas forestales tropicales más importantes de México (PIEDRA, 2016). En 2012, un grupo de menonitas de la colonia 4 Banderas (municipio de Bacalar), el otro productor de soja de Quintana Roo, fue denunciado penalmente por la PROFEPA (Procuraduría Federal de Protección al Ambiente), por provocar un incendio forestal en cerca de 100 hectáreas para destinarlas a agricultura dentro de la reserva Balam Ka'ax (PROFEPA, 2019).

Nos hemos limitado a señalar la deforestación habida sólo en aquellos espacios vinculados al cultivo de soja, en los que si bien los menonitas han sido los actores principales de dicho proceso, también han contribuido a él los ejidatarios que han rentado sus tierras para ser desmontadas por aquéllos, así como los productores privados (entre ellos grandes empresas). Esto ha sido posible por la inacción del gobierno, el cual, a pesar de las escazas acciones emprendidas, ha mantenido, como señala TORRES (2018), una relación de complicidad con los menonitas. Este es un claro ejemplo del uso del mecanismo de autoridad y relaciones sociales planteado en la teoría del acceso, donde se señala que el acceso (legal e ilegal) al estado y otras autoridades permite el control y beneficio de los recursos (RIBOT y PELUSO, 2009). 


\section{CONCLUSIONES}

La reciente expansión de la soja en la península de Yucatán ha dado lugar a un proceso de reconversión productiva y cambio en el uso del suelo, motivado por ventajas agronómicas del grano, elevada rentabilidad, cuantiosos subsidios gubernamentales, oferta de semillas GM y creciente demanda de sus productos finales (carne y huevo). Aunque no puede saberse con exactitud en qué medida la superficie cultivada con soja se conforma por tierras anteriormente dedicadas a maíz, pastizales o selva tropical, el trabajo de campo realizado reveló que en Campeche ha prevalecido la sustitución del maíz y la deforestación, mientras que en Yucatán la soja se ha expandido a costa de los pastizales de la región Oriente y del maíz en el Cono Sur, sin descartar cierto grado de deforestación. En Quintana Roo, la destrucción de la selva ha sido el principal mecanismo de expansión. Esta situación se corresponde con una tendencia a nivel mundial, en la que los bosques tropicales están siendo eliminados para cultivar granos, principalmente soja, lo que representa un gran desafío para la mitigación del cambio climático (HERRERO et al., 2009). Los efectos de la expansión de la soja en la península de Yucatán, la segunda mayor área forestal de América Latina (después del Amazonas), y región con la mayor diversidad biológica, ecológica y cultural de México, ya han dejado en ella lo que WEIS (2013) denomina "huella ecológica", causante no sólo de problemas medio ambientales, sino socio-económicos y de salud (humana y animal).

La teoría del acceso en la que basamos la investigación permitió identificar a los agentes que se han beneficiado del control de los recursos del complejo soja en la península, los procesos o mecanismos de acceso utilizados y las redes de poder conformadas. Encontramos que los mecanismos usados han sido el acceso al capital, tecnología, conocimientos, mercados, autoridad y relaciones sociales, existiendo un elevado grado de complementación e interdependencia entre ellos. Su utilización ha permitido la conformación de una elite de productores y agro-empresas, principales beneficiarios del complejo, quienes han podido ganar, controlar y mantener el acceso a los recursos. Una de sus efectos ha sido la gran concentración de tierras por parte de dichos agentes, basada en su renta a los pequeños productores (ejidatarios), confirmando así lo señalado por la teoría del acceso, en cuanto a que la ausencia de derechos legales o de propiedad no son obstáculo para el control de los recursos, si se cuenta con la habilidad de acceder a ellos a través de ciertos mecanismos (RIBOT y PELUSO, 2009; PELUSO y RIBOT, 2020). Es decir, que los derechos (legales) no son ni una condición necesaria ni suficiente para beneficiarse de los recursos.

Dicho control de las tierras y la expansión de la agricultura mecanizada han sido elementos claves de la consolidación del modelo de producción industrial de la soja en la península. En la medida en la que se ha ido expandiendo la frontera agrícola, lo ha hecho la renta de tierras por parte de los pequeños productores, quienes carecen de los mecanismos de acceso necesarios para emprender un cultivo tan costoso e intensivo en tecnología como es la soja, situación que se repite en diversos países de Sudamérica (MCKAY y COLKE, 2016; CÁCERES, 2014; REBORATTI, 2010). En el caso de México, no se trata de una desposesión de sus tierras, ya que los acuerdos de renta son voluntarios, como tampoco existe un cambio en el régimen de tenencia de la tierra o estructura agraria, ya que los ejidatarios conservan el título de propiedad de sus tierras, sin embargo, quedan excluidos de la actividad productiva agrícola, inherente a su condición de campesinos.

El caso empírico de la soja en la península ejemplifica la importancia de analizar los mecanismos de acceso y control de los recursos, la apropiación y distribución de beneficios, la conformación de redes de poder y los procesos de exclusión que todo ello conlleva, esperando contribuir así al actual debate en torno a la teoría del acceso. 


\section{BIBLIOGRAFÍA}

BERG, B. (2009): Qualitative Research Methods for the Social Sciences. Toronto: Allyn and Bacon. BOHN, T., CUHRA, M., TRAAVIK, T., SANDEN, M., FAGAN., J. y PRIMICERIO, R. (2014): "Compositional differences in soybeans on the market: Glyphosate accumulates in Roundup Ready GM soybeans", Food Quemistry 153: 207-215.

CÁCERES, D. (2014): “Accumulation by dispossesion and socio-environmental conflicts caused by the expansion of agribusiness in Argentina”, Journal of Agrarian Change 15 (1): 116-147.

CARRERA, H. (2017): "Empresarios y menonitas continúan siembra de soja transgénica", La Jornada Maya, 28 de agosto.

CASTILLO, C. (2017): "Menonitas devastan la selva Bacalarense", Novedades de Quintana Roo, 18 de julio.

CCMSS (CONSEJO CIVIL MEXICANO PARA LA SILVICULTURA SOSTENIBLE) (2019): Superficie sembrada de soja en la península de Yucatán. Disponible en: http://132.248.14.102/layers/CapaBase:cultivosoja peninsula [Consulta: 24/09/2019].

DUPUY, J., DURÁN, R., GARCÍA, G., ARELlANO, J., ACOSTA, E., MÉNDEZ, M. y ANDRADE, M. (2015): “Conservation and Use". En Islebe, G., Calmé, S., León, J. y Schmook, B. (Eds.), Biodiversity and Conservation of the Yucatán Peninsula. Switzerland: Springer International Publishing.

ECHÁNOVE, F. (2016): "La expansión del cultivo de la soja en Campeche, México: Problemática y perspectivas", Anales de Geografía 36 (1): 49-69.

ECHÁNOVE, F. (2017): "Subsidies for Price Risk in Grains: The Case of Maize in Mexico", Journal of Iberian and Latin American Research 23 (1): 46-61.

ECHÁNOVE, F. (2018): "Reconversión productiva en Yucatán, México: de maíz y pastizales a soja”, Papeles de Geografía 64: 181-197.

ELlIS, E., ROMERO, J., URIEL, I., PORTER, L. y ELLIS, P. (2017): "Private property and Mennonites are major drivers of forest cover loss in central Yucatan Peninsula, Mexico", Land Use Policy 69: 474-484.

ENTREPRENEUR (2017): "Qué es Agrotech y cómo lo puede aprovechar". Disponible en: https://www.entrepreneur.com/article/300517 [Consulta: 23/08/2017].

ESTOS DÍAS (2019): "La comunidad menonita extiende su frontera agrícola echando la selva abajo", 28 de mayo.

EZQUERRO, A. (2016): "Poisoned, Dispossessed and Excluded: A Critique of the Neoliberal Soy Regime in Paraguay", Journal of Agrarian Change 16 (4): 684-692.

FAO (ORGANIZACIÓN DE LAS NACIONES UNIDAS PARA LA ALIMENTACIÓN Y LA AGRICULTURA) (2019): International Prices. Disponible en: https://fpma.apps.fao.org/giews/food-prices/tool/public/\#/dataset/international [Consulta: 23/08/2019].

FOLEY, J. (2011): "Can we feed the world and sustain the planet?", Scientific American, Noviembre.

GEOCOMUNES (2019): Superficie sembrada con soja en la Península de Yucatán. Disponible en: http://132.248.14.102/layers/CapaBase:cultivosoja_peninsula [Consulta: 12/09/2019].

GFW (GLOBAL FOREST WATCH) (2019): Disponible en: https://www.globalforestwatch.org/map/country/MEX/ [Consulta: 2/09/2019].

GÓMEZ, I. (2016): "A honey-sealed alliance: mayan beekeepers in the Yucatan peninsula versus transgenic soybeans in Mexico's last tropical forest", Journal of Agrarian Change 16 (4): 728736.

GOOGLE EARTH PRO (2019): Disponible en: https://www.google.com/earth/ [Consulta: 3/07/2019].

GRAIN (2014): “2.4-D soy: waging war on peasants”. Disponible en: http://www.grain.org/article/entries/ [Consulta: 7/08/2018]. 
HALL, D., HIRSCH., P. y LI, T. (2011): Powers of exclusion. Singapore: National University of Singapore Press.

HANSEN, A. (2018): "Meat consumption and capitalist development: the meatification of food provision and practice in Vietnam", Geoforum 93: 57-68.

HERNÁNDEZ, S. (2017): "Señalan menonitas a grupos "liberales” en Quintana Roo", El Universal, 11 de septiembre.

HERRERO, M., THORNTON, P., GERBER, P. y REID, R. (2009): "Livestock, livelihoods and the environment: understanding the trade-offs", Current Opinion in Environmental Sustainability 1: 111-120.

HERRERO, M., WIRSENIUS, S., HENDERSON, B., RIGOLOT, C., THORNTON, P., HAVLÍK, P. y GERBER, P. (2015): "Livestock and the environment: What have we learned in the past decade?", Annual Review of Environment and Resources 40: 177-202.

KELLY, J. (2019): "Village-scale reserves in the forest frontier regions of Chenes and Calakmul, Mexico", Journal of Land Use Science: 1-17.

LEGUIZAMÓN, A. (2016): "Environmental Injustice in Argentina: Struggles against Genetically Modified Soy", Journal of Agrarian Change 16 (4): 684-692.

MACHOVINA, B., FEELEY, K. y RIPPLE, W. (2015): "Biodiversity conservation: The key is reducing meat consumption", Science of the Total Environment 536: 419-431.

MASON, R. (2013): "Glyphosate: destructor of human health and biodiversity". Disponible en: http://www.uccs.mx/ [Consulta: 30/07/2018].

MCKAY, B. y COLQUE, G. (2016): "Bolivia's soy complex: the development of "productive exclusión”, The Journal of Peasant Studies 43 (2): 583-610.

MILGROOM, J. y RIBOT, J. (2020): "Children of Another Land: Social Disarticulation, Access to Natural Resources and the Reconfiguration of Authority in Post Resettlement", Society and Natural Resources 33 (2): 184-204.

MUÑOZ, G. (2018): “Transgénicos: no es no", La Jornada, 12 de mayo.

NADERIFAR, M., GOLI, H. y GHALJAEI, F. (2017): "Snowball Sampling: A Purposeful Method of Sampling in Qualitative Research", Strides in Development of Medical Education 14 (3): 1-6.

PELUSO, N. y RIBOT, J. (2020): "Postscript: A Theory of Access Revisited", Society and Natural Resources 33 (2): 300-306.

PELUSO, N. y LUND, C. (2011): "New frontiers of land control: Introduction", The Journal of Peasant Studies 38 (4): 667-681.

PIEDRA, M. (2016): "El cultivo de soja transgénica en Quintana Roo: estudio de caso Ejido Salamanca, Bacalar", Tesis de Maestría, El Colegio de la Frontera Sur.

PORTER, L., SÁNCHEZ, M. y ELLIS, E. (2008): "La conformación del paisaje y el aprovechamiento de los recursos naturales por las comunidades mayas de La Montaña, Hopelchén, Campeche", Investigaciones Geográficas 66: 65-80.

PROFEPA (PROCURADURÍA FEDERAL DE PROTECCIÓN AL AMBIENTE) (2017): "Afectaciones ambientales de comunidades menonitas en Coahuila, Campeche y Quintana Roo", Disponible en: https://www.gob.mx/profepa/prensa/ [Consulta: 22/09/2019].

REBORATTI, C. (2010): "Un mar de soja: la nueva agricultura en Argentina y sus consecuencias", Revista de Geografía Norte Grande 45: 63-76.

RENDÓN, J. y DZUL, R. (2017): "Glyphosate Residues in Groundwater, Drinking Water and Urine of Subsistence Farmers from Intensive Agriculture Localities: A Survey in Hopelchén, Campeche, Mexico", International Journal of Environmental Research and Public Health 14 (6): 2-13.

RIBOT, J. (1998): “Theorizing Access: Forest Profits along Senegal's Charcoal Commodity Chain”, Development and Change 29: 307-341.

RIBOT, J. y PELUSO, N. (2009): “A Theory of Access”, Rural Sociology 68 (2): 158-181.

SADER (SECRETARÍA DE AGRICULTURA Y DESARROLLO RURAL) (2019): "Incentivo para Administración de Riesgos de Precios (Coberturas)". Disponible en: https://www.gob.mx/agricultura [Consulta: 19/03/2019]. 
SIAP (SERVICIO DE INFORMACIÓN AGROALIMENTARIA Y PESQUERA) (2019): Producción Agrícola Anual. Disponible en: https://www.gob.mx/siap/ [Consulta: 19/08/2019].

SUÁREZ, P. (2005): "Migración y salud en una comunidad rural del estado de Campeche", Tesis de Maestría, CINVESTAV Mérida.

TORRES, G., FERNÁNDEZ, J. y GÓMEZ, C. (2018): "La Jurisdicción Agraria y los Derechos Humanos de los Pueblos Indígenas y Campesinos en México". México: Fundación para el Debido Proceso.

TORRES, G. (2018): "Nosotros decimos Ma': la lucha contra la soja transgénica y la rearticulación de la identidad maya en la península de Yucatán", The Journal of Latin American and Caribbean Anthropology 23 (2): 262-280.

UH, J. (2013): "Organización social de los menonitas de Salamanca, Quintana Roo", Tesis de Licenciatura, Universidad de Quintana Roo.

USDA (DEPARTAMENTO DE AGRICULTURA DE ESTADOS UNIDOS) (2019): “Oilseeds: World Markets and Trade". Disponible https://apps.fas.usda.gov/psdonline/circulars/oilseeds.pdf [Consulta: 7/09/2019].

VILLANUEVA, R., ECHAZARRETA, C., GONZÁLEZ, D., ROUBIK, D. y MOGUEL, Y. (2014): "Transgenic soybean pollen (Glycine max L.) in honey from the Yucatan península, Mexico", Scientific Reports 4: 1-4.

WEIS, T. (2013): The ecological hoolprint: The global burden of industrial livestock. New York y London: Zed Books.

WHO (WORLD HEALTH ORGANIZATION) (2015): "Evaluation of five organophosphate insecticides and herbicides". IARC Monographs 12.

\section{ENTREVISTAS DIRECTAS}

ANTONIO (2018). Ejido de Huntochac, 23/05.

BRUNO (2018). Abastecimientos de la empresa Crío, 4/06.

CARLOS (2018). Asesor Agropecuario, 28/05.

CHEN (2019). Técnico agrícola de SEDAPRE, 7/04.

HANS (2017). Productor de la colonia Las Flores, 23/05.

HERNÁN (2018). Empresa Proteínas y Oleicos, 23/05.

KAREN (2019). Agricultor de la colonia El Temporal, 29/03.

LAURO (2019). Centro de Apoyo al Desarrollo Rural de Dzibalchén, 4/04.

LOYD (2019). Encargado de comercialización, colonia Nuevo Progreso, 5/04.

MORA (2019). Técnico agrícola de SEDAPRE, 7/04.

ORIOL (2019). Centro de Apoyo al Desarrollo Rural de Hopelchén, 3/04.

PETER (2019). Agricultor de la colonia Nuevo Progreso, 30/03.

SAMUEL (2019). Agricultor de la colonia Nuevo Progreso, 30/03.

WISE (2016). Productor de Nuevo Progreso, 29/03. 